\title{
Nutraceutical emulsion containing valproic acid (NE-VPA): a drug delivery system for reversion of seizures in zebrafish larvae epilepsy model
}

\author{
Daniela Agustina Feas ${ }^{1}$ Daniela Edith Igartúa ${ }^{1}$ María Natalia Calienni ${ }^{1}$. \\ Carolina Soledad Martinez ${ }^{1} \cdot$ Marina Pifano $^{2} \cdot$ Nadia Silvia Chiaramoni $^{1}$. \\ Silvia del Valle Alonso ${ }^{1} \cdot$ María Jimena Prieto $^{1}$
}

Received: 29 December 2016 / Accepted: 19 February 2017

(C) The Korean Society of Pharmaceutical Sciences and Technology 2017

\begin{abstract}
Valproic acid (VPA) is an antiepileptic drug, which is currently used in neurodegenerative diseases. However, a high dose is required to obtain a therapeutic effect. Long-chain polyunsaturated fatty acids (PUFAs), such as omega 3 and omega 6 , are efficient complements in treatments for neurological diseases. Previous studies have reported that a dietary supplement containing PUFAs together with the administration of antiepileptic drugs significantly reduces the frequency of seizures. Based on this, the main goal of this work was to obtain a complex based on VPA encapsulation in an oil/water $(\mathrm{o} / \mathrm{w})$ nutraceutical emulsion (NE) enriched with PUFAs for oral administration. Besides, encapsulation of VPA might reduce its dose and increase its therapeutic effect. In order to study its effect, we used a zebrafish larvae model of induced epileptiform behavior with the proconvulsant drug pentylenetetrazol (PTZ). Results have shown that when $100 \mu \mathrm{M}$ VPA and fatty acids were combined in the NE (NE-VPA), the epileptiform behavior of PTZ-treated zebrafish larvae decreased significantly. Additionally, morphological changes, hepatotoxicity, lethality and heart rate were studied. Despite the fact that a high dose of VPA exerted a cardiotoxic effect, this was no longer detected after addition of this drug in the NE. This treatment exerted a significant antiepileptic effect and did not result in highly toxic or lethal effects. In order
\end{abstract}

María Jimena Prieto

jprieto@unq.edu.ar

1 Laboratorio de Biomembranas, Departamento de Ciencia y Tecnología, Universidad Nacional de Quilmes, GBEYB. IMBICE, CCT-LA PLATA, CONICET, Bernal, Buenos Aires, Argentina

2 Laboratorio de Oncología Molecular, Departamento de Ciencia y Tecnología, Universidad Nacional de Quilmes, Bernal, Buenos Aires, Argentina to develop an improved pharmaceutical treatment, and considering that all the components used are FDA approved for consumption, the NE-VPA selected might be easily incorporated into clinical trials.

Keywords Epilepsy · Valproic acid · Emulsion · Oral drug delivery $\cdot$ Toxicity $\cdot$ Zebrafish

$\begin{array}{ll}\text { Abbreviations } \\ \text { AED } & \text { Antiepileptic drug } \\ \text { bpm } & \text { Beats per minute } \\ \text { CNS } & \text { Central nervous system } \\ \text { DMSO } & \text { Dymethilsulfoxide } \\ \text { dpf } & \text { Days post-fertilization } \\ \text { GABA } & \text { Gamma-aminobutyric acid } \\ \text { hpf } & \text { Hours post-fertilization } \\ \text { hpi } & \text { Hours post-incubation } \\ \text { MEB } & \text { Model of epileptiform behavior } \\ \text { NE } & \text { Nutraceutical emulsion } \\ \text { NE-VPA } & \text { Nutraceutical emulsion with valproic acid } \\ \text { O/W } & \text { Oil in water } \\ \text { OY } & \text { Opaque yolk } \\ \text { PG } & \text { Propylene glycol } \\ \text { PTZ } & \text { Pentylenetetrazol } \\ \text { PUFAs } & \text { Polyunsaturated fatty acids } \\ \text { SL } & \text { Soy lecithin } \\ \text { VPA } & \text { Valproic acid }\end{array}$

\section{Introduction}

Epilepsy is a chronic disease characterized by neurological disorders that leave a brain predisposition to generate recurrent seizures. This may cause neurobiological, psychological and psychiatric consequences. This disease 
affects $1-2 \%$ of the world's population and the seizures occur unpredictably and periodically, as a consequence of an abnormal electrical activity of neuronal cells. The initial seizure episodes are thought to result from an increase in excitatory neurotransmitters (such as glutamate) and a decrease in the inhibitory neurotransmitter gamma-aminobutyric acid (GABA) (Fisher et al. 2005; Siebel et al. 2011; Rahn et al. 2014).

Antiepileptic drugs (AEDs) are meant to reduce the frequency and/or severity of epileptic seizures in epileptic patients. These drugs treat the epileptic symptoms but not their cause. Usually, AEDs act by preventing the propagation of the discharge to neighboring normal cells (Medscape).

Valproic acid (VPA) has been extensively used as a major AED as well as to exert neuroprotective effects. This drug acts through different pathways from the central nervous system and is used to treat all forms of seizures. Although VPA is generally well tolerated, a high therapeutic dose is required and adverse cognitive side effects are associated with it. It has been reported that VPA induces marked impairments in abstract thinking, imagination, memory, and mathematic reasoning, as well as pancreatitis, teratogenesis and acute hepatic failure in human patients. For these reasons, VPA is contraindicated in pregnant women and young children (Rahn et al. 2014; Balasubramanian et al. 2015) .

Long-chain polyunsaturated fatty acids (PUFAs), such as $\omega$ three and $\omega$ six, may be efficient in an important number of psychiatric and neurological disorders, in particular neurodegenerative diseases. Previous studies have reported that a dietary supplement containing PUFAs, mainly wthree, together with AEDs can alleviate and/or reduce the frequency of epileptic seizures in epileptic patients. This is probably because it has been discovered that these fatty acids increase seizure thresholds and diminish inflammatory mediators, which are increased in these patients (Schlanger et al. 2002; Mazza et al. 2007).

In order to reduce the VPA dose and increase the therapeutic effect, we proposed oral co-administration of VPA combined with $\omega$ in nutraceutical emulsion (NE). As delivery systems, emulsions must increase the solubility and permeability of the drug, prevent hydrolysis or early enzymatic degradation, deliver the drug controllably and facilitate its transport through the intestinal system to increase its bioavailability (Mazza et al. 2007; Lee et al. 2013; Gumpricht et al. 2014).

Zebrafish have been recently proposed as an in vivo model for seizure studies. It has been reported that exposure to pentylenetetrazol (PTZ), a GABA receptor antagonist, induces epileptic-like seizures in zebrafish larvae. Furthermore, Afrikanova et al. (2013) validated this model using a larval locomotor assay as a rapid screening in assessing the anticonvulsant or proconvulsant activity of different drugs; it has also been used for neurogenesis studies due to its greater ability to generate new neurons rather than mammals (Kim et al. 2010; Stewart et al. 2012; Afrikanova et al. 2013). Studies have also reported that zebrafish show epileptiform behavior under treatment with PTZ and that pretreatment with VPA suppresses these adverse effects in the locomotor activity (Lee et al. 2010).

Based on all mentioned before, the aim of this work was to obtain a complex based on VPA encapsulation in an oil/ water $(\mathrm{o} / \mathrm{w})$ nutraceutical emulsion (NE-VPA) enriched with omega 3 and 6 ( $\omega 3$ and $\omega 6)$ fatty acids. Encapsulation of this drug might improve its bioavailability and reduce its secondary effects. The components selected for the emulsion's preparation were canola oil, cod liver oil, vitamin E, Tween 80, soy lecithin (SL) and propylene glycol (PG). Several mixtures with different surfactant proportions were analyzed, and the formulation selected was then characterized by light scattering. Finally, the NE-VPA antiepileptic effect was studied in PTZ-treated zebrafish larvae.

\section{Materials and methods}

\section{Materials}

Cod liver oil, canola oil, vitamin E, Tween 80 and SL, all of which were suited for human intake, were acquired in the local pharmacy. PG was from BioPack (Zárate, Buenos Aires, Argentina). Commercial solution of VPA (Logical ${ }^{\circledR}$ IVAX) and PTZ were donated by Albertina Moglioni from the Chemistry and Drug Metabolism Institute (IQUIMEFA-CONICET) (Buenos Aires, Argentina). Dimethylsulphoxide (DMSO) was from J. T. Baker (Buenos Aires, Argentina) and sodium carboxymethylcellulose was from Sigma-Aldrich (New York, USA). E3 medium was prepared in our laboratory with $5 \mathrm{mM} \mathrm{NaCl}, 0.17 \mathrm{mM}$ $\mathrm{KCl}, 0.33 \mathrm{mM} \mathrm{CaCl}$ and $0.33 \mathrm{mM} \mathrm{Mg}_{2} \mathrm{SO}_{4}$, all of them from BioPack. Ultra Turrax (UT) was an IKA T25 digital model, with rotor S25-20 NK-186 (Labortechnik, Wasserburg, Germany) and WMicrotracker was from Design Plus, SRL (Buenos Aires, Argentina).

\section{Animals}

A breeding stock of heterogeneous wild type zebrafish was inbred in the laboratory aquarium. Sexually mature adult zebrafish (8-12 months) were maintained at $28.0 \pm 1.0^{\circ} \mathrm{C}$ in aquaria with a $14 \mathrm{~h}$ light $-10 \mathrm{~h}$ dark cycle (lights on $=$ Zeitgeber Time Zero). Zebrafish were fed with dry fakes (TetraMin $\mathrm{PRO}^{\circledR}$ ) three times a day and nauplius larvae of Artemia once a day ad libitum. The water in the aquaria was aerated and maintained at $\mathrm{pH} 7.0-8.0$. In this 
study, embryos refer to zebrafish prior to hatching ( $0-3$ day post-fertilization (dpf)), while larvae refer to post-hatching animals (over $3 \mathrm{dpf}$ ).

For embryo production, a ratio of three females and two males were crossed in plastic mesh traps the night before the spawning day to prevent egg cannibalism. In this species, spawning begins with the first light of the day, when males are placed together with females. Embryos were obtained from natural mating, and all embryos and larvae used in these experiments were reared at $28.5^{\circ} \mathrm{C}$ on a 14/10 h light/dark cycle in conditioned E3 medium and $50 \mathrm{ppb}$ methylene blue to inhibit fungal growth. Only fertilized eggs in good condition were selected for further treatment; the others were discarded. The characteristics of eggs were determined with a stereomicroscope (Leica Zoom 2000, Wetzlar, Germany) (Kimmel et al. 1995; Prieto et al. 2014).

\section{Ethics statement $* *$}

All animal procedures were performed in strict accordance with the National Institutes of Health guidelines for animal care and maintenance. The study protocols were approved by the Institutional Animal Care Committee of the National University of Quilmes (CE-UNQ 2/2014) (Buenos Aires, Argentina).

\section{PTZ-treated zebrafish larvae. Development of a model of epileptiform behavior (MEB)}

Zebrafish constitute an attractive model system to study drug toxicity and security as well as to study brain disorders. Moreover, the zebrafish genome is strikingly similar to humans. According to this, $70 \%$ of the protein-coding human genes are related to genes found in the zebrafish (Siebel et al. 2011; Lee et al. 2013).

\section{Experimental design}

A 1 M PTZ stock solution was dissolved in distilled water (Lee et al. 2010). The stock solution was then diluted in E3 medium to the final following concentrations: $1.25,2.50$, 5.00 and $10.00 \mathrm{mM}$ in $\mathrm{E} 3$ medium per well.

At $1 \mathrm{dpf}$, three unhatched embryos were transferred into each well of a 96-well plate containing $250 \mu$ of PTZ different concentrations and incubated at $28^{\circ} \mathrm{C} .16$ wells were used for each treatment.

\section{Measurement of locomotor activity}

The spontaneous movement was studied 10 min post-incubation with the different PTZ drug concentrations at $5 \mathrm{dpf}$. This activity was measured for $15 \mathrm{~min}$. The system used to study zebrafish movements (WMicrotracker, Design Plus SRL, Argentina) is based on an infrared beam arrangement that detects light refraction through the zebrafish body, essentially as described elsewhere (Prieto et al. 2014). Animals were placed in 96-well microplates and subjected to illumination with three infrared beams per well $(100 \mu \mathrm{m}$ wide and $880 \mathrm{~nm}$ wavelength each). A transient fluctuation in the signal is generated when larvae move across the light beam and is then received by a phototransistor array. Output light signals were digitalized by a multichannel ADC system at a sample rate of 10 samples/sec and 10 bit resolution. Data were acquired with an IBM-PC connected via RS232 and processed by software programmed in MSVisual Basic. Signal activity events (defined as the times that larvae crossed through the infrared beams) were calculated in real time by detecting small fluctuations in the signal received. Variations greater than $3 \%$ in the signal received (empirically determined threshold) were considered as activity events. Swimming activity was calculated summing up the number of activity events for $15 \mathrm{~min}$. Data are reported as the average activity event recorded for each beam pair \pm SEM (Lee et al. 2010; Prieto et al. 2012, 2014).

\section{Nutraceutical emulsion (NE)}

\section{Preparation of $N E$}

NE was prepared with a mix of two oils: cod liver oil and canola oil, as previously reported by Igartúa et al. (2015). In this previous work, different combinations of oils/surfactants/co-surfactants were tested and the more stable and with smaller size combination was selected. However, this formulation resulted in high toxicity in zebrafish larvae due to its high amount of Tween 80. Because of this, in the present work, we reduced the amount of this surfactant (Igartúa et al. 2015).

Taking this into account, a mixture of surfactants and co-surfactants was used (Smix). Smix were developed by varying the ratios between the surfactant (Tween 80) and co-surfactants (SL and PG). The SL:PG:Tween 80 mixtures were 1:1:0.006 (Smix 1), 1:1:0.015 (Smix 2) and 1:1:0.030 (Smix 3). Oil mixture and Smix were used in the same proportion.

\section{Characterization of NE: particle size distribution}

The particle size distribution was determined by light scattering (Malvern Mastersizer 2000E; Malvern Instruments Ltd, UK) (Marsanasco et al. 2011). Simultaneously, monitoring was performed by optical microscopy (Leica DMI6000B; Leica Microsystems, GmbH, Wetzlar, Germany) coupled with a digital camera (Leica DC100, Leica Microsystems, GmbH). 
De Brouker mean diameter (D4.3), Sauter mean diameter (D3.2), and the mean size of $90 \%$ of the particles (d0.9) were determined. D4.3 and D3.2 are the mean diameters of the measured distributions in volume and surface, respectively. D4.3 is a parameter sensitive to large particle size, while D3.2 is sensitive to small particle size. Therefore, an increase in D4.3 but not in D3.2 may be related to an aggregation process. $\mathrm{d} 0.9$ is an average of $90 \%$ of the particle population size (Marsanasco et al. 2011).

Stable formulations with small particle size and low concentration of Tween 80 were selected to combine them with VPA.

\section{NE combined with VPA (NE-VPA)}

Smix 2 was selected for the formulation (see "Preparation and selection of NE" section) and it was prepared with and without VPA (NE-VPA and NE, respectively). In NEVPA, an aliquot of VPA was mixed with the Smix and the oil for $1 \mathrm{~min}$ at 10,000 rpm using the UT disperser. Water was added dropwise to the mix for $3 \mathrm{~min}$ at $20,000 \mathrm{rpm}$. During all the agitation process, the sample was kept on ice to avoid an increase in temperature. In NE, PG was added instead of VPA.

\section{Antiepileptic effect}

At $1 \mathrm{dpf}$, three unhatched embryos were transferred into each well of a 96-well plate containing E3 medium and incubated at $28^{\circ} \mathrm{C}$. At $5 \mathrm{dpf}$, the following treatments were tested: (i) 25,50 and $100 \mu \mathrm{M}$ of VPA in NE-VPA, (ii) NE without VPA, (iii) 25,50 and $100 \mu \mathrm{M}$ VPA (iv) control (untreated larvae). Larvae were incubated with treatments during $1 \mathrm{~h}$ at $28^{\circ} \mathrm{C}$. After incubation, $2.50 \mu \mathrm{l}$ of PTZ $(2.50 \mathrm{mM})$ was added in order to induce epileptiform behavior (see "Development of the animal model: MEB" section) (Lee et al. 2010). 10 min later, spontaneous locomotor activity was measured as previously described. 16 wells were used for each treatment. The experimental design is shown in Fig. 1.

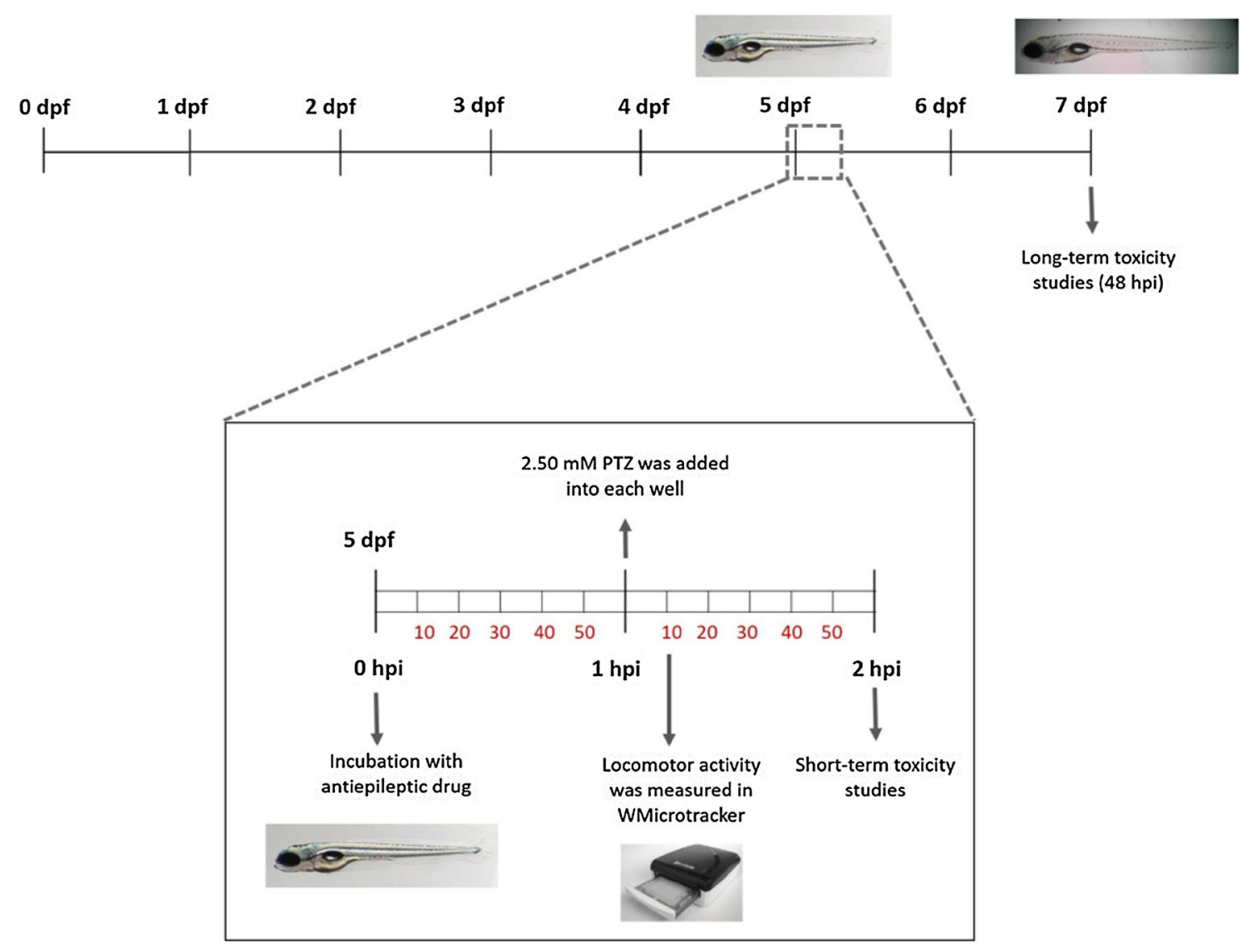

Fig. 1 Experimental design: time line. Incubation times of antiepileptic and proconvulsant drugs. In vivo studies are also described 


\section{Toxicity}

\section{Short-term toxicity}

Short-term toxicity was studied at $2 \mathrm{~h}$ post-incubation (hpi). At $1 \mathrm{dpf}$, unhatched embryos were transferred into each well of a six-well plate containing E3 medium and incubated at $28^{\circ} \mathrm{C}$. At $5 \mathrm{dpf}$, treatments were tested. Control and experimental zebrafish larvae were individually transferred to a depression slide with sodium carboxymethylcellulose and placed under a trinocular microscope Nikon SMZ800. Larvae were photographed with a Microsoft camera and analyzed with the Image J program (free software) to determine possible morphological effects produced by the different treatments. This assay was performed in five larvae per group for each time point. The morphological parameters under study were: eye area, rostro-caudal length and spinal cord length measurements, swim bladder, arching of the body, presence of tissue ulceration or pericardial edema. The same photographs also allowed analyzing liver and yolk. Morphological parameters and phenotypic identification of zebrafish larvae are shown in Fig. 2 (Bar-Ilan et al. 2009; He et al. 2013).

Possible lethal effect was studied in 20 larvae per group for each time point by counting the dead larvae under the microscope.

\section{Long-term toxicity}

Long-term toxicity was studied at $48 \mathrm{~h}$ post-incubation (hpi). Morphological changes, liver and yolk were analyzed. The experimental process was the same as that detailed in "Short-term toxicity" section. Also, as VPA overdose can cause heart block, heart rate after treatments was assessed to study possible cardiotoxic effects. The heart rate was determined by counting the number of beats every $15 \mathrm{~s}$ in a recorded video and then reported as beats per minute (bpm). Experiments were performed three times in five larvae per group for each time point (Prieto et al. 2012).

\section{Statistical analysis}

Data are presented as mean \pm standard (SEM). Statistical analysis was performed by One Way ANOVA, followed by Dunnett's post-test. For non-normally distributed data, or when homoscedasticity was not supported, Kruskal-Wallis test followed by Dunn's post-test was performed. PRISM 6 Version 6.0 was used to conduct all statistical analyses. Only values with $\mathrm{p}<0.05$ were accepted as significant.

\section{Results and discussion}

\section{Preparation and selection of $\mathrm{NE}$}

Emulsion, whose composition was previously detailed, was prepared with different Smix. The corresponding particle size distribution obtained for each formulation is presented in Fig. 3.

For all formulations, we observed D4.3 $>$ D $3.2>\mathrm{d} 0.9$ (Fig. 3; Table 1). This revealed the presence of aggregation processes. Taking into account the values obtained, Smix 1 showed the highest aggregation process, reflected in the difference between d0.9 and D4.3. Besides, Smix 3 presented less aggregation, which is related to the amount of Tween 80 in the surfactant mix (Igartúa et al. 2015). The same results were observed when formulations were monitored by optical microscopy (data not shown).

Figure 3 shows that all the formulations presented monomodal populations with maxima in $1 \mu \mathrm{m}$ approximately. When comparing the surface and volume distributions, we observed that Smix 1 presented one surface population and two volume populations, reflecting aggregation
Fig. 2 Morphology and phenotypic identification. Morphological measurements studied (above), zebrafish larvae at 5 dpf (a) and visual morphology of zebrafish (b) (below)

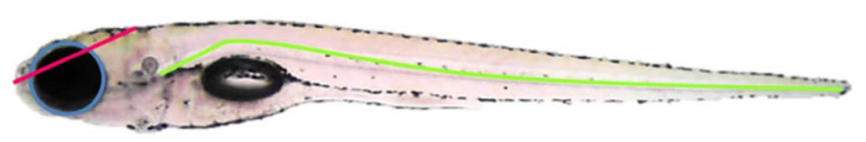

Eve area

Rostrocaudal Length

Spinal Cord Length

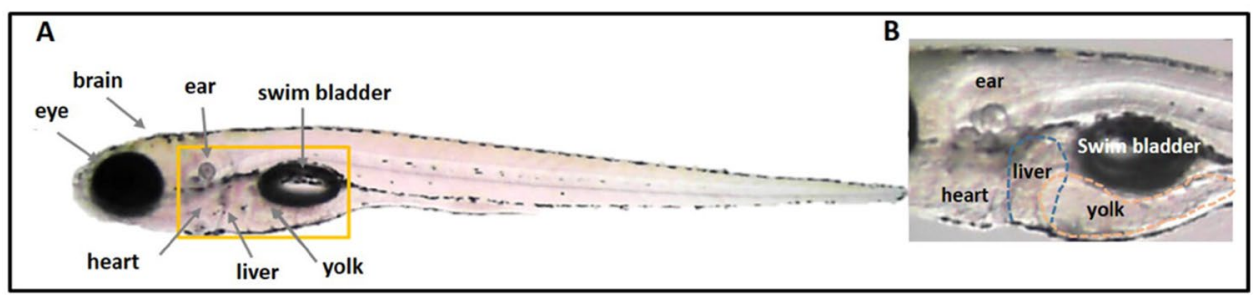



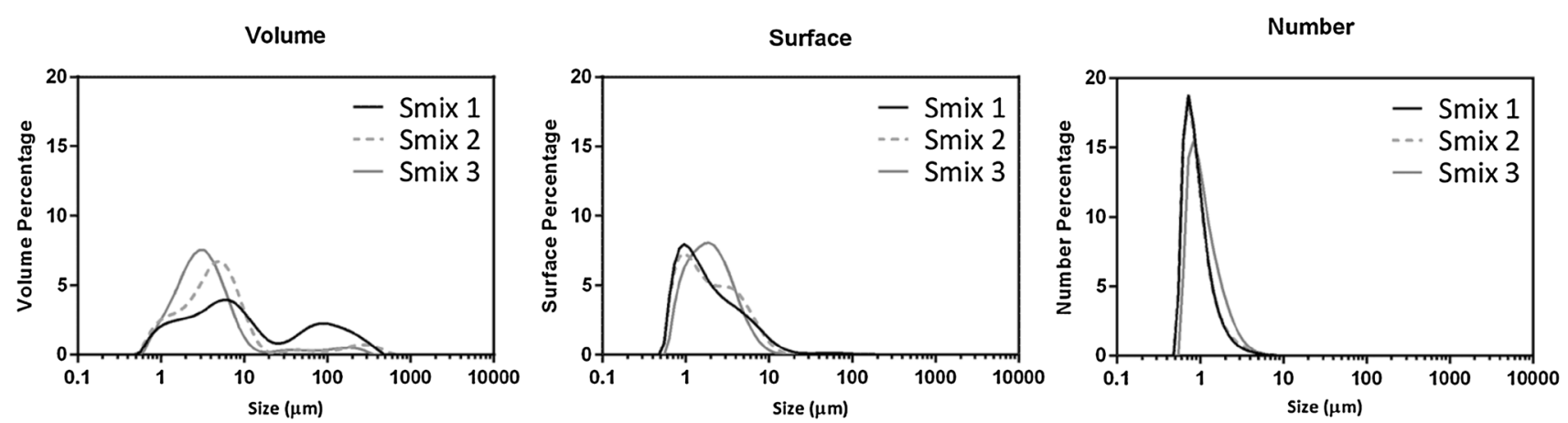

Fig. 3 Particle size distribution in volume, surface and number. Particle size distribution of the emulsion containing different Smix was determined by light scattering Malvern Mastersizer 2000E. Simultaneously, monitoring was performed by optical microscopy (Leica
DMI6000Bm) coupled with a digital camera. De Brouker mean diameter (D4.3), Sauter mean diameter (D3.2) and the mean size of $90 \%$ of the particles (d0.9) were determined

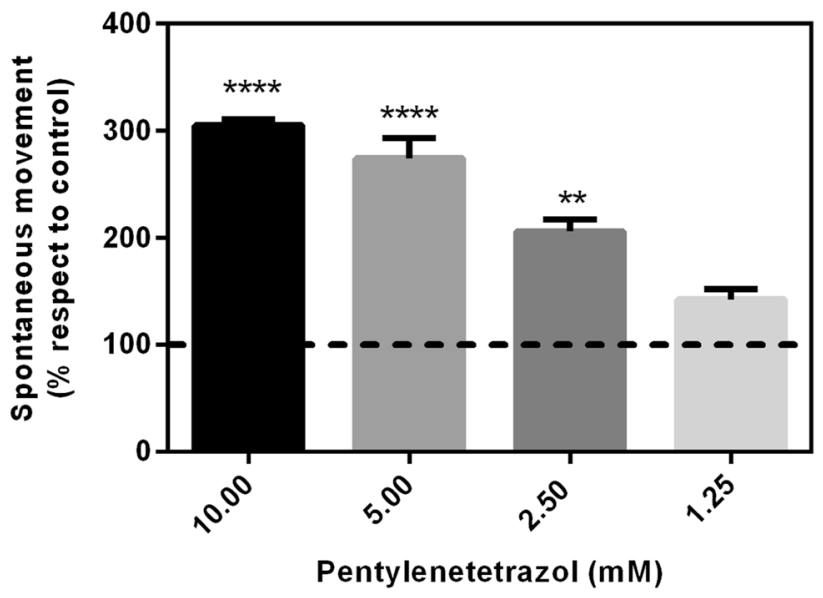

Fig. 4 Locomotor Activity of MEB. Percentage of MEB's spontaneous movement regarding control larvae at $5 \mathrm{dpf}$. Spontaneous movement of control larvae represents $100 \%$ of the locomotor activity. Results are shown as mean \pm SEM $(n=16)$. Statistical analysis was performed by Kruskal-Wallis test and Dunn's post-test $(* * \mathrm{p}<0.01$, $* * * * \mathrm{p}<0.0001)$

amount of the synthetic component (Tween 80 ), which was even more stable.

\section{Development of the animal model: MEB}

Epileptiform seizures were induced in zebrafish larvae by the proconvulsant drug PTZ. Locomotor activity was quantified at $5 \mathrm{dpf}$ and compared to the control (untreated larvae). Concentrations of 1.25, 2.50, 5.00 and $10.00 \mathrm{mM}$ of PTZ were tested. Results are shown in Fig. 4.

Locomotor activity was dependent on PTZ concentration, this means that higher drug concentrations induced a major increase in spontaneous movement respect to the control larvae. This hyperactivity represents an epileptiform behavior, which appeared immediately after the 
Fig. 5 Antiepileptic effect of treatments in MEB. Spontaneous movement of treated larvae with NE-VPA, NE and VPA with $25 \mu \mathrm{M}$ of VPA (a), $50 \mu \mathrm{M}$ of VPA (b) and $100 \mu \mathrm{M}$ of VPA (c) at $5 \mathrm{dpf}$. Results are shown as mean \pm SEM $(n=16)$. Statistical analysis was performed by One Way ANOVA test followed by Dunnett's post-test (a), and Kruskal-Wallis test followed by Dunn's post-test (b and c) $(* \mathrm{p}<0.05, * * \mathrm{p}<0.01, * * * \mathrm{p}<0.001, * * * * \mathrm{p}<0.0001)$. MEB represents $100 \%$ of spontaneous movement. Significant differences represented by *implies that spontaneous movement differed from MEB's, while significant differences represented by $\bullet$ shows a difference in spontaneous movement between treatments

administration of high concentrations of PTZ (5.00 and $10.00 \mathrm{mM})$. In agreement with that observed in previous studies (Lee et al. 2010), larvae exhibited fast and involuntary movements in a circular pattern throughout drug exposure.

The lowest drug concentration that induced epileptiform behavior was $2.50 \mathrm{mM}$. Thus, this concentration was used in further assays.

From now on in this work $2.50 \mathrm{mM}$ PTZ-treated zebrafish larvae will be referred to as model of epileptiform behavior (MEB).

\section{Study of the antiepileptic effect}

In order to study the antiepileptic effect of the treatments, locomotor activity was quantified at $5 \mathrm{dpf}$ and compared to MEB's locomotor activity.

In Fig. 5, treatments with only VPA showed small differences in spontaneous movement respect to MEB. On the other hand, treatments with NE-VPA reduced significantly the epileptiform behavior when were compared to MEB. This effect was further observed with $100 \mu \mathrm{M}$ NE-VPA. Also, treatments with NE decreased larvae spontaneous movement. Even though VPA reduced epileptiform behavior when it was administered alone, the reduction exerted by NE-VPA was much more significant. Particularly, with $100 \mu \mathrm{M}$ treatments there was a huge difference between NE-VPA and VPA and also between NE and VPA. This means that VPA transported by the emulsion triggered a better effect than VPA by itself.

Considering the above discussion, $100 \mu \mathrm{M}$ NE-VPA could be the most suitable system for the reversion of epileptiform behavior.

\section{Toxicity studies}

\section{Short-term toxicity studies (2 hpi)}

None of the treatments resulted in lethal effect at 2 hpi. Besides, there were no morphological changes in larvae when the different treatments were administered (data not shown).
A

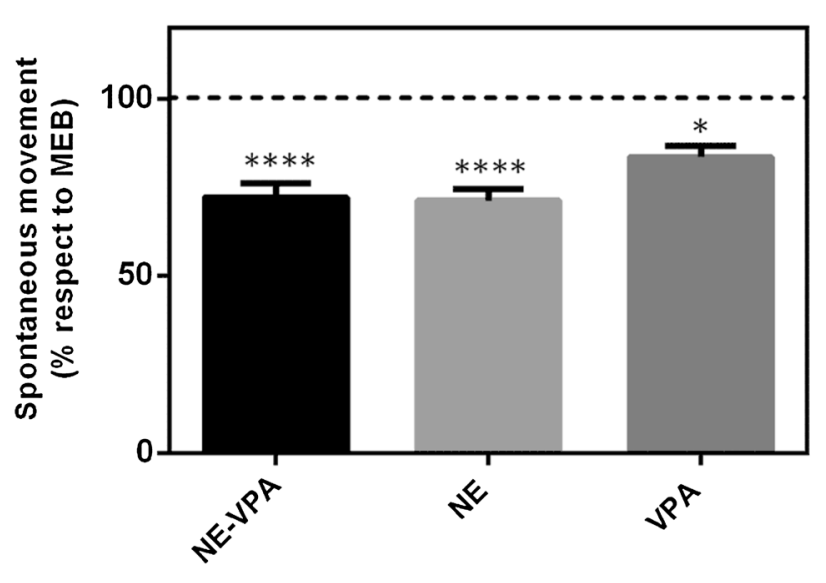

B $\quad 50 \mu \mathrm{M}$ VPA

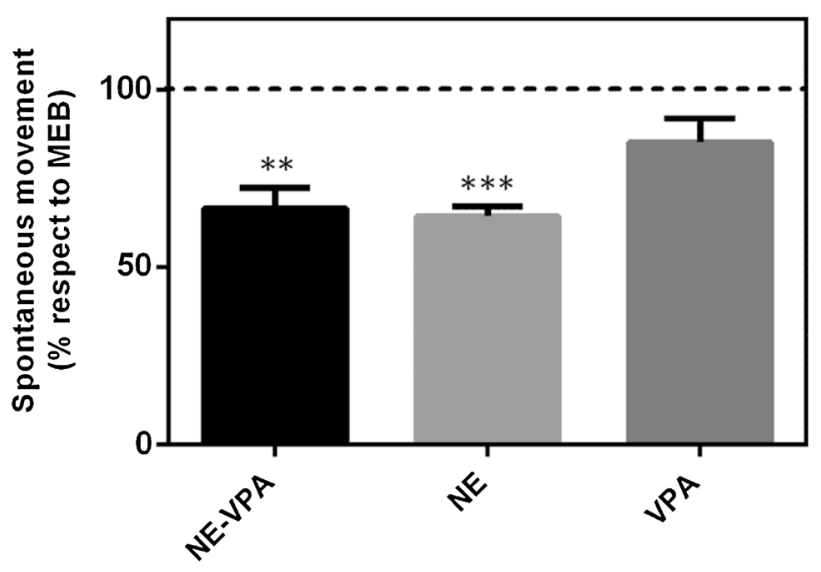

C

$100 \mu \mathrm{M}$ VPA

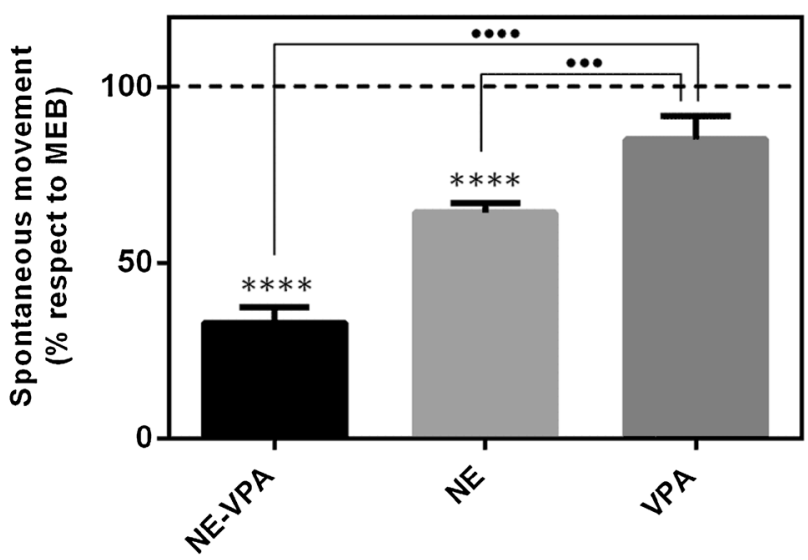

Long-term toxicity studies (48 hpi)

Measures of eye area, rostrocaudal length and spinal cord length of treated larvae did not significantly differ from those of control larvae, but MEB did show an increase in eye area (data not shown). Also, MEB showed an 
uninflated or even a completely loss of the swim bladder, as well as tissue ulceration; in some of these larvae, pericardial edema and a slight arching of the body was also observed. Regarding hepatotoxicity effects, MEB's liver became dark (Fig. 6). He et al. (2013) reported that this liver condition corresponded to liver damage (He et al. 2013). Therefore, PTZ might be considered an hepatotoxic drug.

On the other hand, a small part of the treated larvae presented a slight arching of the body and, when treated with VPA only, some larvae also showed an uninflated swim bladder. Furthermore, larvae opaque yolk (OY) was studied as a sublethal endpoint in NE-VPA treatments (Bar-Ilan et al. 2009). This condition was observed in all these treatments in a dose-dependent manner (Fig. 6).

Moreover, only MEB and treated larvae with $25 \mu \mathrm{M}$ NE and $100 \mu \mathrm{M}$ VPA exhibited a cardiotoxic effect. $100 \mu \mathrm{M}$ VPA treatment and MEB showed a significant decrease in the heart rate respect to control larvae, while $25 \mu \mathrm{M}$ NE treatment showed a significant increase in it. This proves that a high dose of VPA by itself could be extremely cardiotoxic. It is worth to distinguish that this effect was not seen when the drug was combined in the emulsion in the same concentration (Fig. 7).

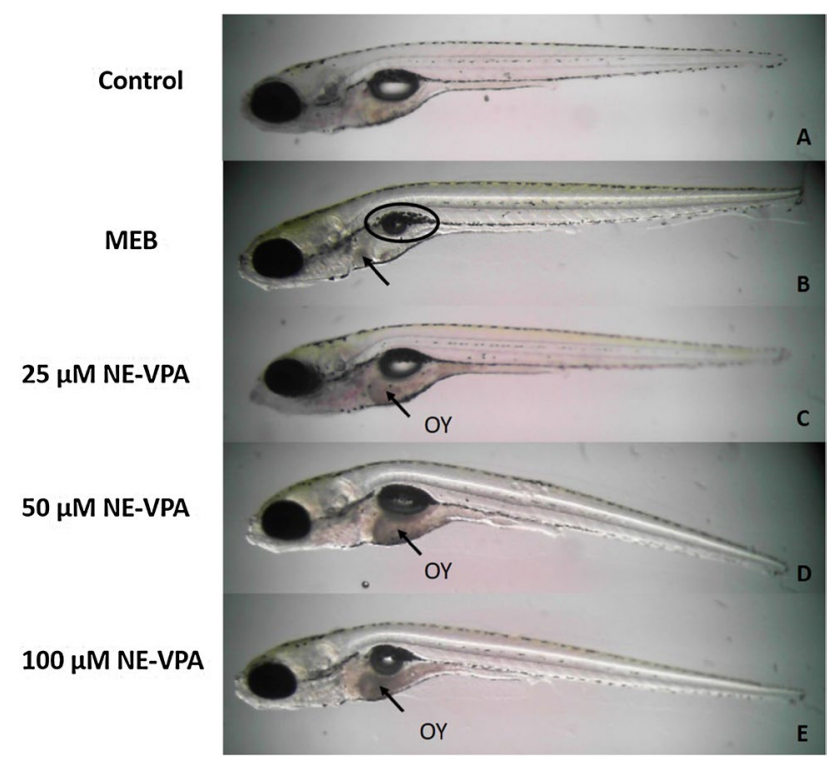

Fig. 6 Long-term Control larvae (a), MEB $(b)$ and treated larvae with $25 \mu \mathrm{M}$ NE-VPA (c), $50 \mu \mathrm{M}$ NE-VPA $(d)$, and $100 \mu \mathrm{M}$ NE-VPA (e). An uninflated swim bladder and a dark liver were observed in MEB. The circle shape and the arrow, respectively, indicate these abnormalities $(b)$. Opaque yolk was observed in 25, 50 and $100 \mu \mathrm{M}$ NE-VPA treatments. Arrows in $c, d$ and $e$ indicate this sublethal point

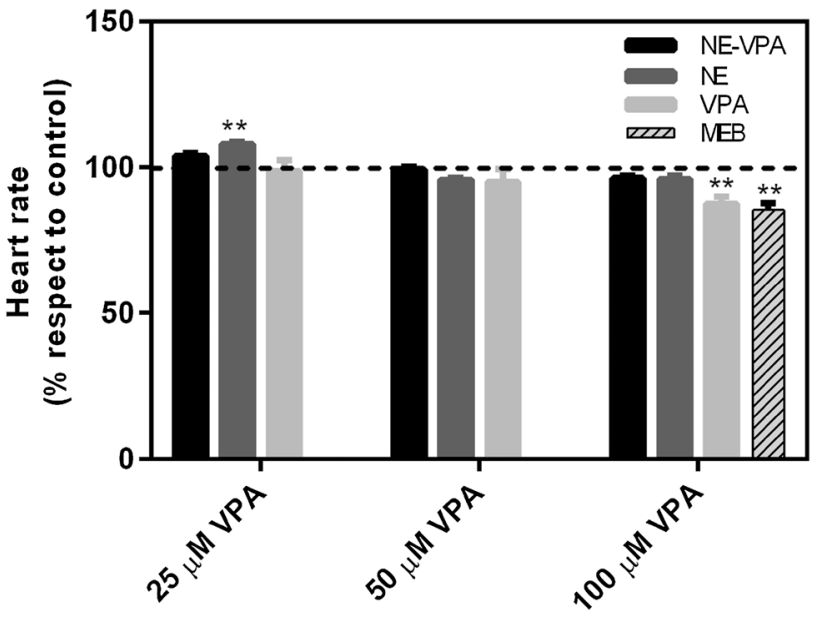

Fig. 7 Heart rate analysis. Heart rate of MEB and treated larvae with NE-VPA, NE and VPA with $25 \mu \mathrm{M}$ of VPA, $50 \mu \mathrm{M}$ of VPA and $100 \mu \mathrm{M}$ of VPA at $48 \mathrm{hpi}(7 \mathrm{dpf})$. Results are shown as mean \pm SEM of five independent measures for each treatment. Statistical analysis was performed by Kruskal-Wallis test and Dunn's post-test, comparing all the samples with control larvae $(* * \mathrm{p}<0.01)$

\section{Conclusions}

In the present study, we designed a drug delivery system with NE that was stable and able to incorporate the antiepileptic drug VPA. Two main issues should be highlighted. First, the treatment with NE-VPA at high dose $(100 \mu \mathrm{M})$ exerted a significant antiepileptic effect, which lead to a complete reversion of the epileptiform behavior. Besides, this treatment did not result in highly toxic or lethal effects. Second, considering that all the components used are FDA approved for consumption, the NEVPA selected might be easily incorporated into clinical trials. This could lead to an improved pharmaceutical treatment. This work could be considered a starting point to address new studies to search for better treatments for neurodegenerative diseases, such as epilepsy.

Acknowledgements This research was supported by a grant from Universidad Nacional de Quilmes, Consejo Nacional de Investigaciones Científicas y Técnicas (CONICET) (PIP214/11) and Ministerio Nacional de Ciencia, Tecnología e Innovación Productiva (MINCYT). María Jimena Prieto, Silvia del Valle Alonso and Nadia Silvia Chiaramoni are members of CONICET, Argentina. Daniela Agustina Feas acknowledge fellowships from Consejo Interuniversitario Nacional (CIN) and from CONICET, Argentina.

\section{Compliance with ethical standards}

Conflict of interest All authors declare that they have no conflict of interest. 


\section{References}

Afrikanova T, Serruys A-SK, Buenafe OE, Clinckers R, Smolders I, de Witte PA, Crawford AD, Esguerra CV (2013) Validation of the zebrafish pentylenetetrazol seizure model: locomotor versus electrographic responses to antiepileptic drugs. PLoS ONE 8(1):e54166

Balasubramanian D, Deng AX, Doudney K, Hampton MB, Kennedy MA (2015) Valproic acid exposure leads to upregulation and increased promoter histone acetylation of sepiapterin reductase in a serotonergic cell line. Neuropharmacology 99:79-88

Bar-Ilan O, Albrecht RM, Fako VE, Furgeson DY (2009) Toxicity assessments of multisized gold and silver nanoparticles in zebrafish embryos. Small 5(16):1897-1910

Fisher RS, Boas WvE, Blume W, Elger C, Genton P, Lee P, Engel J (2005) Epileptic seizures and epilepsy: definitions proposed by the International League Against Epilepsy (ILAE) and the International Bureau for Epilepsy (IBE). Epilepsia 46(4):470-472**

Gumpricht E, Rockway S (2014) Can $\omega$-3 fatty acids and tocotrienolrich vitamin $\mathrm{E}$ reduce symptoms of neurodevelopmental disorders? Nutrition 30(7):733-738

He J-H, Guo S-Y, Zhu F, Zhu J-J, Chen Y-X, Huang C-J, Gao J-M, Dong Q-X, Xuan Y-X, Li C-Q (2013) A zebrafish phenotypic assay for assessing drug-induced hepatotoxicity. J Pharmacol Toxicol Methods 67(1):25-32

Igartúa DE, Calienni MN, Feas DA, Chiaramoni NS, Valle Alonso SD, Prieto MJ (2015) Development of nutraceutical emulsions as risperidone delivery systems: characterization and toxicological studies. J Pharm Sci 104(12):4142-4152

Kim Y-H, Lee Y, Lee K, Lee T, Kim Y-J, Lee C-J (2010) Reduced neuronal proliferation by proconvulsant drugs in the developing zebrafish brain. Neurotoxicol Teratol 32(5):551-557

Kimmel CB, Ballard WW, Kimmel SR, Ullmann B, Schilling TF (1995) Stages of embryonic development of the zebrafish. Dev Dyn 203(3):253-310

Lee Y, Kim D, Kim YH, Lee H, Lee CJ (2010) Improvement of pentylenetetrazol-induced learning deficits by valproic acid in the adult zebrafish. Eur J Pharmacol 643(2-3):225-231

Lee SH, Kim HR, Han RX, Oqani RK, Jin DI (2013a) Cardiovascular risk assessment of atypical antipsychotic drugs in a zebrafish model. J Appl Toxicol 33(6):466-470
Lee SH, Kang JW, Lin T, Lee JE, Jin DI (2013b) Teratogenic potential of antiepileptic drugs in the zebrafish model. BioMed Res Int $20131-6$

Marsanasco M, Márquez AL, Wagner JR, Alonso SdV, Chiaramoni NS (2011) Liposomes as vehicles for vitamins E and C: an alternative to fortify orange juice and offer vitamin $\mathrm{C}$ protection after heat treatment. Food Res Int 44(9):3039-3046

Mazza M, Pomponi M, Janiri L, Bria P, Mazza S (2007) Omega-3 fatty acids and antioxidants in neurological and psychiatric diseases: an overview. Prog Neuropsychopharmacol Biol Psychiatry 31(1):12-26

Medscape http://emedicine.medscape.com/article/1187334-overview

Neslihan Gursoy R, Benita S (2004) Self-emulsifying drug delivery systems (SEDDS) for improved oral delivery of lipophilic drugs. Biomed Pharmacother 58(3):173-182

Pichot R, Spyropoulos F, Norton I (2010) O/W emulsions stabilised by both low molecular weight surfactants and colloidal particles: the effect of surfactant type and concentration. J Colloid Interface Sci 352(1):128-135

Prieto MJ, Gutierrez HC, Arévalo RA, Chiaramoni NS, del Valle Alonso S (2012) Effect of risperidone and fluoxetine on the movement and neurochemical changes of zebrafish. Open J Med Chem 2:129-138

Prieto MJ, del Rio Zabala NE, Marotta CH, Bichara D, Simonetta S, Chiaramoni NS, del Valle Alonso S (2014) G4. 5 Pamam dendrimer-Risperidone: biodistribution and behavioral changes in in vivo model. J Nanomed Biother Discov 4(1):1-11

Rahn J, Bestman J, Josey B, Inks E, Stackley K, Rogers C, Chou C, Chan S (2014) Novel vitamin K analogs suppress seizures in zebrafish and mouse models of epilepsy. Neuroscience 259:142-154

Schlanger S, Shinitzky M, Yam D (2002) Diet enriched with omega-3 fatty acids alleviates convulsion symptoms in epilepsy patients. Epilepsia 43(1):103-104

Siebel AM, Piato AL, Capiotti KM, Seibt KJ, Bogo MR, Bonan CD (2011) PTZ-induced seizures inhibit adenosine deamination in adult zebrafish brain membranes. Brain Res Bull 86(5):385-389

Stewart AM, Desmond D, Kyzar E, Gaikwad S, Roth A, Riehl R, Collins C, Monnig L, Green J, Kalueff AV (2012) Perspectives of zebrafish models of epilepsy: what, how and where next? Brain Res Bull 87(2):135-143 